\title{
Role of social interactions during digital game-based learning in Science Education: A systematic review
}

\author{
Pey-Yng Low \\ Ngee Ann Polytechnic, Singapore
}

\begin{abstract}
Digital games have been used for teaching science subjects; however, merely playing games does not guarantee that learning will take place. Educators need to scaffold the gameplay experience and integrate other instructional methods into the process to enhance and ensure learning. Social constructivism is one such approach. Although there are a number of studies on game-based learning, they focus more on the effectiveness of learning or the classification of learning outcomes rather than the mode of social interactions in game-based learning. A systematic review was carried out to identify the different modes of social interactions and their impact on digital game-based learning in science education. Five modes of social interactions were identified; face-to-face conversation between students, group discussion, online/virtual collaboration, teacher-facilitated classroom discussion and answering of questions by teachers. Social interactions enhanced learning through collaborative sense making, promoting learner motivation, enabling scientific reasoning and providing instructional support. These form a basis for educators to design productive social interactions for digital game-based learning in science education.
\end{abstract}

Keywords: Game-based learning, social learning, social constructivism, science education

\section{Background}

Digital games have been shown to benefit science education by enhancing conceptual learning, developing high- level cognitive skills, and shaping student identity, attitudes and scientific habits of mind (Clark, 2011; Steinkeuhler, 2008; Chee, 2012). However, learning may not be meaningful or effective if games are used as a standalone instructional method (Tobias, 2007) and gameplay alone does not guarantee that learning will occur (Tennyson, 2008). Social interactions in game-based learning can support learning by providing opportunities to share knowledge, clarify misconceptions, and promote reflection as well as problem-solving (Mikropolour, 2011; Shih, 2010). Studies have shown that social behaviours such as social comparison and help-seeking can also enhance learning and engagement (Vasalou, 2017; Clark, 2011). Furthermore, meaningful interactions with classmates, teachers or virtual collaborators allow students to construct knowledge and acquire conceptual understanding (Dalgarno, 1996).

Research studies on game-based learning in the sciences generally focus on the acquisition of disciplinary knowledge. There is little analysis focusing on the mode of social interactions and their impact on learning. The effectiveness of social interactions would be dependent on strategies utilised and the situational contexts (van der Meij, 2010). Hence, there is a need for educators to be aware of possible strategies for the effective implementation of social constructivist approaches in digital game-based learning.

A systematic review was carried out to address the following questions:

1. In what ways do digital game-based learning in science education provide opportunities for students to have meaningful social interactions?

2. How do social interactions influence learning and engagement during digital game-based learning in science education? 


\section{Method}

\section{Data collection and analysis}

Databases and search strategy

A search was conducted for peer-reviewed journals from 2009 to 2019 in the following educational research databases: Academic Search Complete, Educational Research Complete and ERIC, available on the University of Melbourne's Library website. Database searches were performed using the following search terms:

- Game-based learning - ("serious games" OR "educational games" OR "game based learning" OR GBL)

- Digital games - (video OR computer OR digital OR online OR virtual)

- Outcomes and impact of playing games - (impacts OR outcomes OR effects OR influence OR performance OR motivation OR engagement OR behavio* OR emotion OR enjoyment OR attitude)

- Science education - (science OR chemistry OR physics OR biology)

- Social interactions - (social* OR interact* OR collaborat* OR cooperat*)

\section{Coding of papers}

Twenty papers meeting the criteria were coded using a data extraction standard used by previous studies that categorised educational games (Connolly, 2012). For mode of social interactions, the categories were constantly refined throughout the process of analysis and finalised after all papers were analysed.

Quality assessment

To assess the quality of papers, paper were given a score of $1-3$ for the following dimensions as described by a previous study (Connolly, 2012); 3 indicates a high score, 2 indicates a medium score and 1 indicates a low score for that criterion.

1. Quality of study design.

1.1. $\quad$ High $=3$, e.g., randomised controlled experiment

1.2. Medium $=2$, e.g., quasi-experimental controlled study

1.3. $\quad$ Low $=1$, e.g., case study, pre-test/post-test design

2. Appropriateness of methods $\&$ analysis used in study.

3. Generalisability of study to the target population with respect to the size and representativeness of sample to this review.

4. Relevance of study for addressing the questions of this review.

5. Reliability of study findings in the answering of study question(s). 


\section{Results}

\section{Papers identified}

The search preliminarily identified 688 citations. After removing duplicates, applying the inclusion and exclusion criteria, and through citation chaining, 20 papers were included in the systematic review (Figure 1).

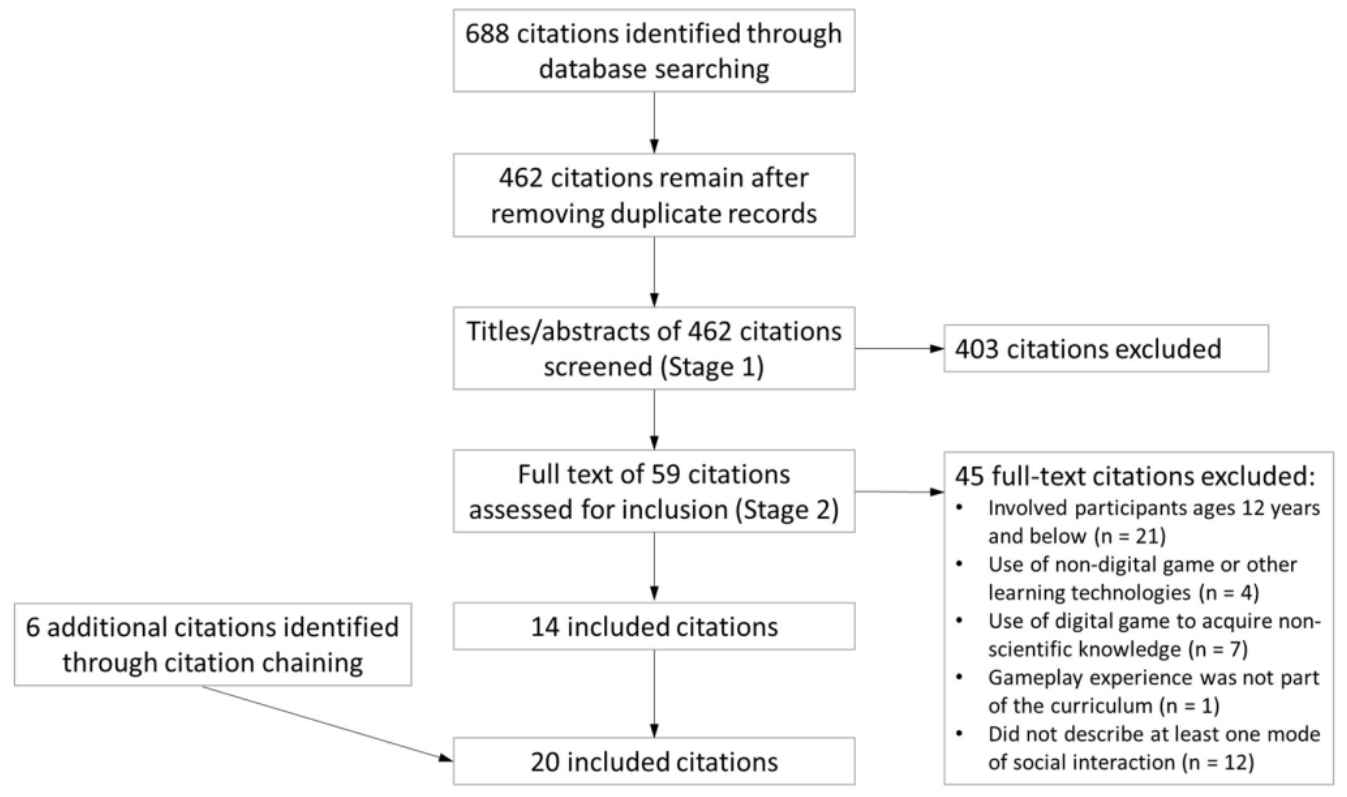

Figure 1. Flow diagram illustrating the identification of included citations

\section{Quality scores of papers}

Twenty papers were given a quality score as described earlier. Scores ranged from $5-15$ with 5 being a low score and 15 being a high score. The mean rating for 20 papers was 9.9 and the modal rating for the papers was 10 . There were 5 papers with a quality score of 8 and lower. The lower scores were due to their study design, as these papers were mainly observational studies, case studies and studies involving pre/post-test design or surveys. The nature of the study design had also affected the scores of other quality criteria due to their sample size, resulting in the lower overall quality score. However, as the focus of this review was on the mode of social interaction during game-based learning, observational data from classroom implementation could provide insights to the effectiveness of social interaction during game-based learning. Thus, all 20 papers were included.

\section{Study design used in papers}

Table 1 shows the number of papers adopting each study design, with quasi-experimental controlled studies being the most common. Of the 20 studies, 9 focused on the role of social interaction in game-based learning. 
Table 1. Focus of study (social interaction) by study design for all papers

\begin{tabular}{lccc}
\hline \multirow{2}{*}{ Study Design } & \multicolumn{2}{c}{$\begin{array}{c}\text { Is social interaction the main } \\
\text { focus of the study? }\end{array}$} & Total \\
\cline { 2 - 3 } & Yes & No & \\
\hline Case study & 1 & 1 & 2 \\
Correlation study & 0 & 1 & 1 \\
Observational study & 2 & 0 & 2 \\
Pre-/post-test design & 1 & 0 & 1 \\
Quasi-experimental controlled study & 2 & 8 & 10 \\
Randomized controlled experiment & 2 & 1 & 3 \\
Survey and interviews & 1 & 0 & 1 \\
& & & \\
Total & 9 & 11 & 20 \\
\hline
\end{tabular}

\section{Game genre}

While there was a range of game genres as described by previous studies (Connolly, 2012; Ke, 2016), papers in this review fell into 4 main categories namely, adventure (8), puzzle (5), role-playing (5) and strategy (2) games (Table 2). It could be that these game genres may be more apt for science education. Games were most commonly designed for learning of physics (10), followed by biology (7) and chemistry (3).

Table 2. Focus of study (social interaction) by game genre for all papers

\begin{tabular}{lccc}
\hline Game Genre & \multicolumn{2}{c}{$\begin{array}{c}\text { Is social interaction the main } \\
\text { focus of the study? }\end{array}$} & Total \\
\cline { 2 - 3 } & Yes & No & \\
\hline Adventure & 4 & 4 & 8 \\
Puzzle & 1 & 4 & 5 \\
Role-playing & 2 & 3 & 5 \\
Strategy & 2 & 0 & 2 \\
Total & 9 & 11 & 20 \\
\hline
\end{tabular}

\section{Mode of social interactions by focus of study (social interaction)}

The modes of social interaction are shown in Table 3. Two or more modes of social interaction were observed in 10 studies. Among the 15 instances of social interaction reported by papers focusing on social interactions, $13(87 \%)$ involved student-student interactions and $2(13 \%)$ involved student-teacher interactions. For papers which did not have a focus on social interaction, among the 16 instances of social interactions, $5(31 \%)$ involved student- student interactions and $11(69 \%)$ involved student-teacher interactions.

Table 3. Focus of study (social interaction) by mode of social interactions

\begin{tabular}{lccc}
\hline Mode of social interactions & \multicolumn{2}{c}{$\begin{array}{c}\text { Is social interaction the main focus } \\
\text { of the study? }\end{array}$} & \multirow{2}{*}{ Total } \\
\cline { 2 - 3 } & \multicolumn{2}{c}{ Yes } & No \\
\hline Student-student interaction & 5 & 3 & 8 \\
Face-to-face conversations between students & 5 & 2 & 7 \\
Group discussion & 3 & 0 & 3 \\
Online/ virtual collaboration & & & \\
Student-teacher interaction & 0 & 7 & 7 \\
Teacher facilitated class discussion & 2 & 4 & 6 \\
Answering of questions by teachers & & & 31 \\
Total & 15 & 16 & \\
\hline
\end{tabular}




\section{Discussion on mode of social interactions}

The papers were categorised based on the mode of social interactions and listed in Table 4 .

Table 4. Overview of citations included in the systematic review

\begin{tabular}{|l|l|}
\hline \begin{tabular}{l} 
Mode of social interactions \\
\hline Student-student interaction
\end{tabular} \\
\hline $\begin{array}{l}\text { Face-to-face conversations between } \\
\text { students }\end{array}$ & $\begin{array}{l}\text { (Barab, 2009; Annetta, 2010; Clark D. B., 2011; Magnussen, 2014; Shute, } \\
\text { 2015; Eaton, 2015; Ye, 2017; Srisawasdi, 2019) }\end{array}$ \\
\hline Group discussion & $\begin{array}{l}\text { Echeverria, 2011; Echeverria A. A., 2012; Echeverria A. B., 2012; } \\
\text { Magnussen, 2014; Chen, 2015; Ye, 2017; Chang, 2017) }\end{array}$ \\
\hline Online/ virtual collaboration & (Hummel, 2011; Eaton, 2015; Annetta, 2010) \\
\hline \multicolumn{2}{|l|}{} \\
\hline Student-teacher interaction & $\begin{array}{l}\text { Chee, 2012; Sadler T. D., 2013; Brom, 2015; Rowe, 2017; Srisawasdi, 2019; } \\
\text { Eastwood, 2013; Sadler T. D., 2015) }\end{array}$ \\
\hline Teacher-facilitated class discussion & (Annetta, 2010; Echeverria, 2011; Clark D. B., 2011; Echeverria A. A., 2012; \\
Echeverria A. B., 2012; Eastwood, 2013)
\end{tabular}

Face-to-face conversations between students

Face-to-face conversations between students during gameplay was the most extensively described mode of social interaction. Students engaged in a range of conversations, from what was happening on their own or others' screens to a discussion regarding the underlying scientific concepts (Shute, 2015). Peer interaction during game- based learning provided an opportunity for collaborative sense making (Barab, 2009; Annetta, 2010; Srisawasdi, 2019; Eaton, 2015). During the gameplay experience, students worked together to problem-solve and eventually achieve a shared conceptual understanding (Barab, 2009). In a game involving genetics, students actively discussed underpinning scientific knowledge to construct Punnett Squares and pedigrees. The discussions enabled students to refine their understanding of genetics (Annetta, 2010). Peer interaction allowed students to discuss the scientific phenomena observed during gameplay. For chemistry, students clarified misconceptions and generated their own knowledge regarding the properties of liquids (Srisawasdi, 2019). Peer assistance behaviours were also commonly observed during the game-based learning experience, allowing students to receive timely clarifications from their classmates regarding scientific concepts (Eaton, 2015).

Peer-to-peer interactions during game-based learning increased student motivation for learning (Clark, 2011; Magnussen, 2014; Ye, 2017; Annetta, 2010). Students were motivated due to the opportunity for social interaction as well as the competitive nature of games. Engaging discussion between students served as a source of motivation for game completion, allowing students to have ample opportunities to review and apply prior learning during the process of repetitive play (Annetta, 2010). When using commercial games to support physical concept construction, students shared their game strategies and achievements, which contributed to energetic group discussions and active participation (Ye, 2017). Student-led competition was also a motivating factor as students compared their achievement status (eg. medals or scores attained) with their peers, celebrating when high scores were obtained (Magnussen, 2014; Clark, 2011). This promoted game replayability as students sought strategies to improve their in-game performance.

Lastly, face-to-face interactions between students provided an opportunity for the explicit articulation of scientific terminologies and reasoning (Eaton, 2015; Magnussen, 2014). Scientific reasoning can have multiple levels of complexity, ranging from "concrete specifications" where student verbally specified solutions without any game- based or scientific-based rationale, "concrete reasoning" where student discussions revolved around game elements and mechanics to "formal scientific reasoning" where student 
discussions involved formal scientific terminology or concepts (Eaton, 2015). By providing students with opportunities for verbal scientific reasoning, pieces of knowledge were organised and students developed coherence in conceptual understanding.

\section{Group Discussion}

There were 7 studies documenting group discussion and in these studies, students were grouped into pairs or small groups during the game-based learning experience. Group discussions brought about learning benefits by enabling collaborative problem solving (Chen, 2015; Chang, 2017), motivation towards learning

(Echeverria, 2011; Ye, 2017), authentic learning through a simulated professional setup (Magnussen, 2014) and deep conceptual understanding by exploring different perspectives (Echeverria A. A., 2012; Echeverria A. B., 2012).

While the benefits from a group-based discussion overlap with that of face-to-face interactions, comparative studies highlighted the advantages of group discussion during game-based learning. A mission synchronisation- based peer-assistance approach was implemented in a study to investigate the role of teambased collaborative learning as opposed to individual learning through gameplay (Chang, 2017). The approach required all the team members to complete the current gaming mission before proceeding to the next mission. Pronounced mutual assistance and collective problem-solving behaviours were observed in the experimental group where the approach was implemented but not in the control group where participants played the game individually. Besides enhancing learning outcomes, the enhanced level of collaboration between team members also improved attitudes towards learning (Chang, 2017). In another study where flipped learning strategies were adopted, cooperative learning and discussion allowed for sharing of information and motivation in the completion of textbook exercises after gameplay (Ye, 2017). Students were also observed to be enthusiastic about sharing their ideas, which contributed to a greater learning efficiency. High levels of motivation persisted throughout the gameplay sessions and students were visibly excited with significant amounts of positive interactions between students (Echeverria, 2011).

Team-based discussions also provided a unique opportunity for gaining deeper conceptual understanding by allowing students to adopt different perspectives during gameplay. In a study involving a game on electrostatics, students were presented with different physical points of views (eg. as the first person or third person) during their gameplay experience. By exploring the problem from multiple perspectives, students gained better conceptual understanding regarding electrostatics (Echeverria A. B., 2012; Echeverria A. A., 2012). This mode of collaborative learning was also adopted in a scientific discovery game that allowed players to develop a quantum computer. Students were introduced to a simulated professional setup, each enacting a different role in a research team of physicists. By relating to specific scientific domains required for their roles, students engaged in complex discussions to share their findings. The gameplay experience was highly motivating for students due to the ability to participate in real science and solve authentic problems (Magnussen, 2014).

While most studies highlighted the advantages of collaborative learning through group discussions, one described the occurrence of unproductive groups due to incompatible personality traits. During the study, members of the group did not work well together, did not like to work with others and were constantly engaged in disagreements. While the implementation of group discussion led to an enhancement of learning experience and promoted collective problem solving, the posttest scores were lower than that of the control group where group discussion was not introduced (Chen, 2015). Hence, collaborative game-based learning may present challenges especially for students with incompatible personality traits or in a class with a highly competitive culture.

\section{Online/ virtual collaboration}

Online collaboration was reported in three studies in the form of in-game text chats (Annetta, 2010), online forum discussions (Eaton, 2015) and collaboration through email communication (Hummel, 2011).

In the first study involving an in-game text chat function, the chat function seemed to be the preferred mode of communication as compared to verbal conversations. Comments in the text chat were collaborative in 
nature, suggesting that students could effectively collaborate via an online medium (Annetta, 2010). Positive outcomes of online collaboration were also documented in a study where students shared gameplay strategies and reflected upon their gameplay experiences on an online forum after the successful completion of their mission (Eaton, 2015). Communication via online forums were more likely to employ formal scientific reasoning as compared to face-to-face interactions. This ascertained the utility of online forums in enabling students to explicitly articulate key disciplinary relationships, allowing students to achieve deep conceptual understanding (Eaton, 2015). While online real-time collaboration appeared to be favoured by students, a prescripted email collaboration with a 'virtual' counterpart drew negative feedback. This mode of collaboration did not involve direct student-student interaction but rather, students received an email from a 'virtual' colleague who took an alternative stance. Students did not find this mode of collaboration effective (Hummel, 2011).

\section{Teacher-facilitated class discussion}

Teacher-facilitated class discussions were described in 7 studies mostly with the intention to reinforce knowledge acquisition related to gameplay experiences (Sadler, 2015; Sadler, 2013; Eastwood, 2013; Brom, 2015; Rowe, 2017). Teachers played a central role in helping students made explicit connections between classroom activities and gameplay experiences to deepen students' understanding (Eastwood, 2013). Students' conceptual understanding was enhanced through a variety of questioning techniques such as the use of "bell ringer" questions to prompt students to recall prior knowledge and the "big picture questions" to emphasise key ideas and stimulate higher order thinking (Eastwood, 2013).

In a study on game-based inquiry learning, teacher-facilitated class discussion inculcated a classroom culture of a community of inquiry where students developed the scientific way of thinking (Chee, 2012). To help develop skills for inquiry-based learning, students were asked to self-evaluate the quality of questions they had proposed in order to recognise the factors contributing to a good scientific question (Chee, 2012). Through this process, students reported greater self-awareness and self-regulation in their thinking, greater curiosity about science and being more critical and discerning regarding textbook knowledge. Another study involving game-transformed inquiry-based learning had also reported improved students' conceptual understanding and promoted their motivation in learning chemistry (Srisawasdi, 2019).

\section{Answering of students' questions by teachers}

Although there were 6 studies that discussed the involvement of teachers in the answering of students' questions during gameplay (Annetta, 2010; Echeverria, 2011; Clark, 2011; Echeverria A. A., 2012; Echeverria A. B., 2012; Eastwood, 2013), there was little elaboration regarding the advantages of this mode of social interaction. One study highlighted the importance of answering questions as the timely facilitation by teachers enabled the linking of prior knowledge to gameplay experiences, allowing for the reinforcement of learning (Annetta, 2010).

\section{Discussion}

As there was limited in-depth analysis of the mode of social interaction during game-based learning in science education, this systematic review explored the various modes of social interactions and their impact in a game- based learning setting. There were more extensive reporting and detailed elaborations on studentstudent interactions, as compared to student-teacher interactions, suggesting a student-centred nature of these publications. The benefits of student-student interactions revolved around three key themes; collaborative learning, motivation for learning as well as the scientific practices and thinking. Collaboration was deemed important for game-based learning in a scientific curriculum as it promoted knowledge construction and deep conceptual understanding (Chen, 2015; Acar, 2006; Frailichm, 2009). A number of studies described the role of face-to-face conversations (between students) in collaborative sense making (Barab, 2009; Annetta, 2010; Eaton, 2015; Srisawasdi, 2019). This suggested that collaborative learning could occur in a student-led manner, even without formalised conditions requiring students to do so. However, when students were placed into groups during the game-based learning experience, the outcomes of collaborative learning became more evident, especially in situations where students were assigned different viewpoints (Magnussen, 2014; Echeverria A. A., 2012; Echeverria A. B., 2012) or if there was a need for interdependence during gameplay 
(Chang, 2017). These findings were consistent with Dillenbourg's (1999) recommendation that the probability of collaborative learning could be enhanced by incorporating conditions to facilitate collaboration (Dillenbourg, 1999). Hence, educators may wish to consider the incorporation of a group component in the design of game-based learning experience, to enhance student learning through collaboration.

Student-student interactions also resulted in heightened motivation towards learning. We observed two sources of motivation arising from social interactions that is, the feelings of relatedness and the opportunity for social comparison. Social interaction was motivating in nature due to the basic psychological need for relatedness (Ryan

R. M., 2006). This was manifested in the form of highly engaging discussions that contributed to the increased game completion and participation in learning activities. (Annetta, 2010; Ye, 2017). The intrinsic need for relatedness was also reported in another study as students synchronised their gameplay experience to foster a sense of group identity (Vasalou A. e., 2017). On the other end of the spectrum, social interaction was also an opportunity for social comparison and this was amplified in the presence of game elements (eg. medals or score system) that promoted in-game competition (Clark D. B., 2011; Magnussen, 2014). As student-student interactions foster learner's motivation, the inclusion of social interaction opportunities in game design (eg. in- game chat function and competitive game elements) and game-based learning experience (eg. group discussions and peer assistance approaches) will be a key consideration when implementing game-based learning.

Scientific dialogue between students, or with teachers, was crucial in the development of scientific reasoning skills and this was exemplified by studies on inquiry-based learning. Past studies have highlighted the importance of inquiry strategies in knowledge construction and how these could lead to an improved understanding of scientific processes and knowledge (Schauble, 1996; National Research Council, 2000). Similar findings were found in this review as game-transformed inquiry-based learning led to an enhancement of conceptual understanding and motivation towards the learning of chemistry (Chee, 2012; Srisawasdi, 2019). Interaction with teachers was found to be key as it allowed students to develop questioning skills and created a community of inquiry where students engaged in active questioning (Chee, 2012). Besides the development of scientific reasoning skills, dialogue between students enabled them to align and express their intuitive understanding of scientific concepts in abstract, or seemingly counterintuitive, language of science (Eaton, 2015). This could also lead to conceptual change as students reconstruct their knowledge and develop coherence in their understanding of science (diSessa, 2004). Interestingly, online forums were found to be a more effective mode of collaboration over face-to-face interaction when it comes to formal scientific reasoning. This was attributed to the reflective nature of online forums and constraints in the traditional classroom, where speaking in formal scientific language was socially prohibitive (Eaton, 2015).

While social interaction between students brings about benefits during game-based learning, teachers play an important role in lesson planning and the facilitation of classroom discussion. The case study on teachers having different implementations of the same curriculum highlighted the central role of teachers in determining the outcome of game-based learning (Eastwood, 2013). There was a varied degree in how teachers made explicit connections between the game and curriculum materials and this would impact the effectiveness of game implementation. There were studies identifying the need for educational games to be integrated with appropriate instructional support in order to yield maximum educational benefits (Tobias, 2007; Sitzmann, 2011; Garris, 2002). Games as a stand-alone learning tool were ineffective as students were unable to understand complex relationships from gameplay experience alone (Garris, 2002). Hence, it is important for teachers to incorporate sound pedagogical practices and instructional support when implementing game-based learning.

\section{Conclusion \& Recommendations}

As games are complex learning environments, students can be overwhelmed by the excessive and multimodal modes of information, the dynamic nature of gameplay and the complexity of task to be performed (Wouters, 2013). Hence, there is a need to implement instructional support to help scaffold students' learning and social constructivist approaches could form the basis of such instructional support. This review identified five modes of social interactions during classroom implementation of game-based learning: face-to-face 
conversation between students, group discussion, online/virtual collaboration, teacher-facilitated classroom discussion and answering of questions by teachers. As each of these modes have differential effects on the learning of science, the effectiveness of incorporating social interactions will also be dependent on the intended outcomes of game-based learning. The following recommendations are proposed for the design of an in-class game-based learning experience to meet the specific educational objectives:

To enhance the acquisition of complex scientific knowledge:

- Incorporate group discussions to facilitate understanding of abstract concepts through collaborative sense making

- Make explicit connections between prior learning, classroom activities and gameplay experience to deepen students' understanding

- Incorporate a debrief session to clarify misconceptions

To facilitate explicit articulation of scientific terminologies or reasoning:

- Provide opportunities for verbal scientific reasoning by incorporating a group component in the design of game-based learning experience

- Facilitate class discussion following gameplay to inculcate a culture of scientific inquiry

- Initiate an online discussion forum for students to reflect on game-based learning experience

To enhance student motivation and in-class participation:

- Incorporate group component during game-based learning experience to enhance their sense of relatedness and identity

- Incorporate friendly competition between groups of students to encourage repetitive play. This allows students to more opportunities to apply their learning.

Besides design considerations during the implementation phase of game-based learning, educators can also consider the incorporation of game elements during the design of educational games. Some possibilities include the incorporation of in-game chat function to facilitate mutual discussion and leader board to promote social comparison behaviours. While social interaction during game-based learning was largely found to be advantageous, there may be situations where non-productive interactions can negate the benefits of gamebased learning. Educators will have to monitor student interactions and intervene if such situations arise. These are important considerations during the planning and implementation of a game-based learning curriculum for science education as social interactions can be engineered into the learning experience to enhance learning outcomes.

\section{Limitations}

The current review has a number of limitations. The review was limited by the databases available to the university, by the search terms used, the journals that were selected and the time period of publications. As social interaction was not usually the main focus of scientific games, papers could have been omitted as a category of search terms was related to social interaction. Hence, citation chaining was also employed to find relevant articles.

\section{References}

Acar, B. (2006). Effect of cooperative learning strategies on students' understanding of concepts in electrochemistry. International Journal of Science and Mathematics Education, 5(2), 349-373.

Annetta, L. A. (2010). Assessing twenty-first century skills through a teacher created video game for high school biology students. Research in Science \& Technological Education, 28(2), 101-114.

Barab, S. A. (2009). Transformational play as a curricular scaffold: Using videogames to support science education. Journal of Science Education and Technology, 18, 305-320.

Brom, C. (2015). Playing educational micro-games at high schools: individually or collectively. Computers in Human Behavior, 48, 224-235.

Chang, S. (2017). Development of an effective educational computer game based on a mission 
synchronization- based peer-assistance approach. Interactive Learning Environment, 25(5), 667-681.

Chee, Y. S. (2012). Becoming chemists through game-based inquiry learning: The case of Legends of Alkhimia. Electronic Journal of e-Learning, 10(2), 185-198.

Chen, C. (2015). The comparison of solitary and collaborative modes of game-based learning on students' science learning and motivation. Educational Technology \& Society, 18(2), 237-248.

Clark, D. B. (2011). Exploring newtonian mechanics in a conceptually-integrated digital game: Comparison of learning and affective outcomes for students in Taiwan and the United States. Computers \& Education, 57, 2178-2195.

Connolly, T. M. (2012). A systematic literature review of empirical evidence on computer games and serious games. Computers \& Education, 59, 661-686.

Crookall, D. (1995). A guide to the literature on simultaion/gaming. In D. a. Crookall, Simulation and gaming across disciplines and cultures (pp. 151-177). Thousand Oaks, CA: Sage.

Dalgarno, B. (1996). Constructivist computer assisted learning: Theory and techniques. Australasian Society for Computers in Tertiary Education. Adelaide: ASCILITE. Retrieved October 20, 2019, from http://www.ascilite.org/conferences/adelaide96/papers/21.html

de Freitas, S. (2006). Learning in immersive worlds. London: Joint Information Systems Committee.

Dickey, M. D. (2005). Engaging by design: How engagement strategies in popular computer and video games can inform instructional design. Educational Technology, 53(2), 67-83.

Dillenbourg, P. (1999). What do you mean by "collaborative learning"? In P. Dillenbourg, Collaborativelearning: Cognitive and Computational Approaches (pp. 1-19). Oxford: Elsevier.

diSessa, A. (2004). Coherence versus fragmentation in the development of the concept of force. Cognitive Science, 28(6), 843-900.

Divjak, B. (2011). The impact of game-based learning on the achievement of learning goals and motivation for learning mathematics - literature review. Journal of Information and Organizational Sciences, 35(1), $15-30$.

Eastwood, J. L. (2013). Teachers' implementation of a game-based biotechnology curriculum. Computers \& Education, 66, 11-24.

Eaton, G. V. (2015). Patterns of physics reasoning in face-to-face and online forum collaboration around a digital game. International Journal of Education in Mathematics, Science and Technology, 3(1), 1-13.

Echeverria, A. A. (2012). Exploring different technological platforms for supporting colocated collaborative games in the classroom. Computers in Human Behaviour, 28, 1170-1177.

Echeverria, A. B. (2012). The atomic intrinsic integration approach: A structured methodology for the design of games of the conceptual understanding of physics. Computers \& Education, 59, 806-816.

Echeverria, A. (2011). A framework for the design and integration of collaborative classroom games. Computers \& Education, 57, 1127-1136.

Ermi, L. (2005). Fundamental components of the gameplay experience: Analysing immersion. Proceedings of DiGRA 2005 conference: Changing Views -Worlds in Play (pp. 15-27). Vancouver, CA: Simon Fraser University.

Frailichm, M. (2009). Enhancing students' understanding of the concept of chemical bonding by using activities provided on an interactive website. Journal of Research in Science Teaching, 46(3), 289-310.

Garris, R. A. (2002). Games, motivation, and learning: A research and practice model. Simulation \& Gaming, $33(4), 441-467$.

Gee, J. P. (2003). What Video Games Have to Teach Us About Learning and Literacy. New York: Palgrave Macmillen.

Hummel, H. G. (2011). Scripted collaboration in serious gaming for complex learning: Effects of multiple perspectives when acquiring water management skills. British Journal of Educational Technology, 42(6), 1029-1041.

Ke, F. (2016). Designing and integrating purposeful learning in game play: a systematic review. Educational Technology Research and Development, 64, 219-244.

Ketelhut, D. J. (2010). A multi-user virtual environment for building and assessing higher order inquiry skills in science. British Journal of Educational Technology, 41, 56-68.

Lederman, L. C. (1992). Debriefing [Special issue]. Simulation \& Gaming, 23(2).

Magnussen, R. (2014). Games as a platform for student participation in authentic scientific research. The Electronic Journal of e-Learning, 12, 259-270.

Mikropolour, T. A. (2011). Educational virtual environments: a ten-year review of empirical research. 
Computers \& Education, 56, 769-780.

National Research Council, N. (2000). Inquiry and the national science education standards: a guide for teaching and learning. Washington, DC: National Academic Press.

O'Neil, H. F. (2005). Classification of learning outcomes: evidence from computer games literature. The Curriculum Journal, 16(4), 455-474.

Reese, H. W. (2011). The learning-by-doing principle. Behavioral Development Bulletin, 11, 1-19.

Rowe, E. (2017). Assessing implicit science learning in digital games. Computer in Human Behavior, 76, 617-630.

Ryan, R. M. (2006). The motivational pull of video games: A self-determination theory approach. Motivation and Emotion, 30, 347-363.

Sadler, T. D. (2013). Game-based curriculum in biology classes: Differential effects among varying academic levels. Journal of Research in Science Teaching, 50(4), 479-499.

Sadler, T. D. (2015). Learning biology through innovative curricula: A comparison of game- and nongame approaches. Science Education, 99(4), 696-720.

Schauble, L. (1996). The development of scientific reasoning in knowledge-rich contexts. Developmental Psychology, 32(1), 102-119.

Shih, J. L. (2010). The influence of collaboration styles to children's cognitive performance in digital problem- solving game "William Adventure": A comparative case study. Computers \& Education, 55(3), 982-993.

Shute, V. J. (2015). Modeling how incoming knowledge, persistence, affective states and in-game progress influence student learning from an educational game. Computers \& Education, 86, 224-235.

Sitzmann, T. (2011). A meta-analytic examination of the instructional effectiveness of computer-based simulation games. Personnel Psychology, 64, 489-528.

Srisawasdi, N. (2019). Implementation of game-transformed inquiry-based learning to promote the understanding of and motivation to learn chemistry. Journal of Science Education and Technology, 28, $152-164$.

Steinkeuhler, C. (2008). Scientific habits of mind in virtual worlds. Journal of Science, 17(6), 530-543.

Subrahmanyam, K. (1994). Effect of video game practice on spatial skills in girls and boys. Journal of Applied Developmental Psychology, 15, 13-32.

Tennyson, R. D. (2008). A conceptual framework for the empirical study of instructional games. In H. F. O' Neil, Computer games and team and individual learning (pp. 39-54). Oxford, UK: Elsevier.

Tobias, S. (2007). What research has to say about designing computer games for learning. Educational Technology, 20-29.

van der Meij, H. (2010). Learning from games: Does collaboration help? British Journal of Educational Technology, 42(4), 655-664.

Vasalou, A. (2017). Digital game-based learning for children with dyslexia: A social constructivist perspective on engagement and learning during group game-play. Computers \& Education, 114, 175192.

Wouters, P. V. (2013). A meta-analysis of the cognitive and motivational effects of serious games. Journal of Educational Psychology, 105(2), 249-265.

Ye, S. (2017). Using commercial video games in flipped classrooms to support physical concept construction. Journal of Computer Assisted Learning, 34, 602-614.

Yee, N. (2006). Motivations for play in online games. CyberPsychology and Behavior, 9(6), 772-775.

Low, P.Y. (2020). Role of Social Interactions during Digital Game-based Learning in Science Education: A Systematic Review. In S. Gregory, S. Warburton, \& M. Parkes (Eds.), ASCILITE's First Virtual Conference. Proceedings ASCILITE 2020 in Armidale (pp. 208-218). https://doi.org/10.14742/ascilite2020.0110

Note: All published papers are refereed, having undergone a double-blind peer-review process.

The author(s) assign a Creative Commons by attribution licence enabling others to distribute, remix, tweak, and build upon their work, even commercially, as long as credit is given to the author(s) for the original creation.

(C) Yng Low, P. 2020 